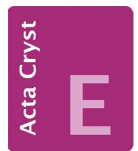

CRYSTALLOGRAPHIC COMMUNICATIONS

ISSN 2056-9890

Received 26 January 2016

Accepted 18 February 2016

Edited by M. Weil, Vienna University of Technology, Austria

Keywords: crystal structure; 1,4,8,11-tetraazaundecane; chloride ligand; trans-meso (RS) conformation; chromium(III) complex; hydrogen bonding; synchrotron radiation.

CCDC reference: 1454582

Supporting information: this article has supporting information at journals.iucr.org/e

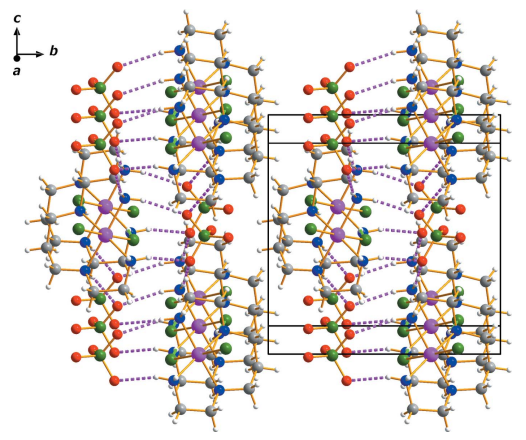

OPEN $\odot$ ACCESS

\section{Crystal structure of trans-dichlorido(1,4,8,11-tetra- azaundecane- $\kappa^{4} N$ )chromium(III) perchlorate determined from synchrotron data}

\author{
Dohyun Moon ${ }^{\mathrm{a}}$ and Jong-Ha Choi ${ }^{\mathrm{b} *}$
}

${ }^{\mathbf{a}}$ Pohang Accelerator Laboratory, POSTECH, Pohang 37673, Republic of Korea, and ${ }^{\mathbf{b}}$ Department of Chemistry, Andong National University, Andong 36729, Republic of Korea. *Correspondence e-mail: jhchoi@anu.ac.kr

The structure of the title complex, $\left[\mathrm{CrCl}_{2}\right.$ (2,3,2-tet) $] \mathrm{ClO}_{4}$ (2,3,2-tet is $1,4,8,11$ tetraazaundecane, $\mathrm{C}_{7} \mathrm{H}_{20} \mathrm{~N}_{4}$ ), has been determined from synchrotron data. The $\mathrm{Cr}^{\mathrm{III}}$ ion is coordinated by the four $\mathrm{N}$ atoms of the 1,4,8,11-tetraazaundecane ligand in the equatorial plane and two chloride ions in an axial arrangement, displaying a slightly distorted octahedral coordination environment. The two $\mathrm{H}$ atoms of the secondary amines are grouped on the same side of the equatorial $\mathrm{N}_{4}$ plane (meso-RS conformation). The $\mathrm{Cr}-\mathrm{N}$ bond lengths range from 2.069 (2) to 2.084 (2) $\AA$, while the mean $\mathrm{Cr}-\mathrm{Cl}$ bond length is 2.325 (2) $\AA$. The crystal structure is stabilized by intermolecular hydrogen-bonding interactions between the primary and secondary amine groups of the 2,3,2-tet ligands, the $\mathrm{Cl}$ ligands and the $\mathrm{O}$ atoms of the perchlorate counter-anion, forming corrugated layers parallel to (010).

\section{Chemical context}

Geometric and conformational isomerism in chromium(III) complexes of linear flexible tetradentate ligands is an interesting field because it has played an important role in extending the concept of stereochemistry. The 1,4,8,11-tetraazaundecane ligand (2,3,2-tet) is a structural isomer of 1,4,7,11-tetraazaundecane (2,2,3-tet). These two ligands have four nitrogen atoms as donor groups and can adopt three different configurations in chromium(III) complexes with two

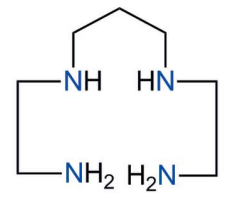

2,3,2-tet

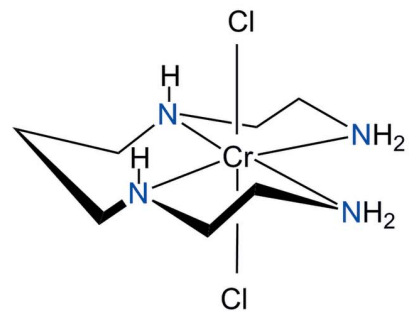

meso-RS conformer
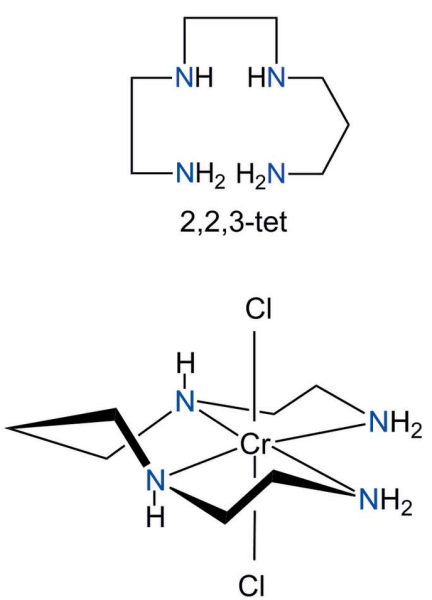

rac-RR/SS conformer
Figure 1

Schematic representation of the 2,3,2-tet and 2,2,3-tet ligands, and two possible conformational isomers of trans $-\left[\mathrm{CrCl}_{2}(2,3,2-\mathrm{tet})\right]^{+}$. 
additional $\mathrm{Cl}$ ligands (Choi et al., 2008a,b). Two conformations of meso-RS or racemic-RR/SS isomers with respect to the orientation of the secondary amine hydrogen atoms in the trans isomer are also possible (Fig. 1). The two hydrogen atoms of the conformers may be on the same side $(R S)$ of the equatorial $\mathrm{N}_{4}$ plane or on opposite sides $(R R / S S)$ of this plane.

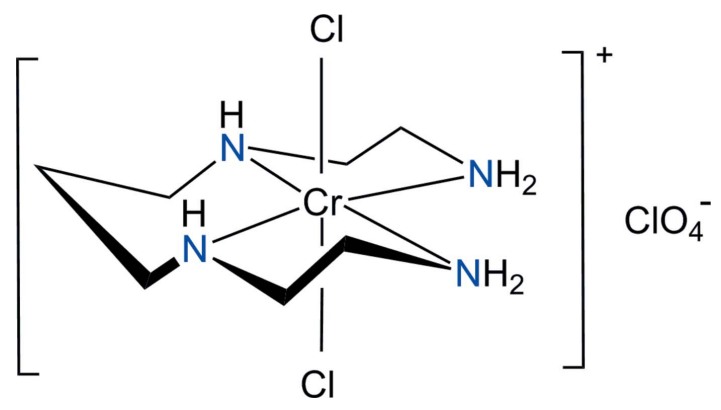

The different symmetries of transition metal complexes allow the determination of their stereochemistry from electronic absorption and infrared spectra. Indeed, infrared and electronic spectroscopic properties often are useful in determining the geometric isomers of chromium(III) complexes with linear tetradentate ligands (House \& Garner; 1966; Kutal \& Adamson, 1973; House \& Yang, 1983; Kirk \& Fernando, 1994). However, it should be noted that the geometric assignments based on spectroscopic studies alone are less conclusive. Both trans and cis isomers of $\left[\mathrm{CrCl}_{2}(2,3,2-\mathrm{tet})\right]-$ $\mathrm{ClO}_{4}$ have been isolated (House \& Yang, 1983; Kirk \& Fernando, 1994). Whereas the crystal structure and spectroscopic properties of the cis- $\beta$-dichloridochromium(III) complexes containing the 2,3,2-tet ligand were reported (Choi et al., 2008b), the trans isomers with any anion have so far not been structurally characterized. The orientation of the secondary amine hydrogen atoms in the metal complexes is also highly relevant for medical application and likely to be a major factor in determining the antiviral activity (Ronconi \& Sadler, 2007; Ross et al., 2012). In order to confirm the orientation of the secondary $\mathrm{N}-\mathrm{H}$ hydrogen atoms of the

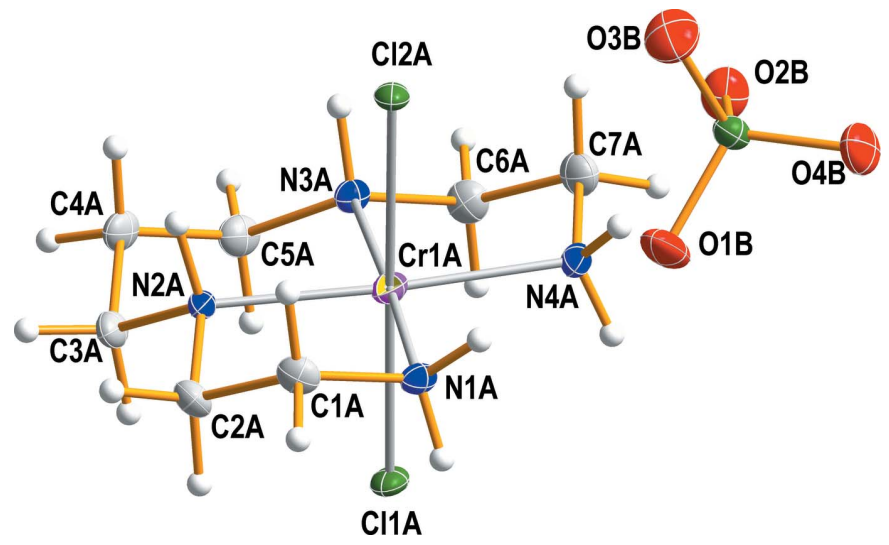

Figure 2

The structures of the molecular components of complex (I), drawn with displacement ellipsoids at the $30 \%$ probability level.
Table 1

Hydrogen-bond geometry $\left(\AA{ }^{\circ}\right)$.

\begin{tabular}{lllll}
\hline$D-\mathrm{H} \cdots A$ & $D-\mathrm{H}$ & $\mathrm{H} \cdots A$ & $D \cdots A$ & $D-\mathrm{H} \cdots A$ \\
\hline $\mathrm{N} 1 A-\mathrm{H} 1 A 1 \cdots \mathrm{O} 2 B^{\mathrm{i}}$ & 0.90 & 2.30 & $3.187(4)$ & 167 \\
$\mathrm{~N} 1 A-\mathrm{H} 1 A 2 \cdots \mathrm{O} 1 B$ & 0.90 & 2.30 & $3.180(4)$ & 164 \\
$\mathrm{~N} 2 A-\mathrm{H} 2 A \cdots \mathrm{Cl} 1 A^{\mathrm{ii}}$ & 0.99 & 2.47 & $3.332(2)$ & 146 \\
$\mathrm{~N} 3 A-\mathrm{H} 3 A \cdots \mathrm{O} 1 B^{\text {iii }}$ & 0.99 & 2.28 & $3.174(4)$ & 150 \\
$\mathrm{~N} 4 A-\mathrm{H} 4 A 1 \cdots \mathrm{O} 2 B$ & 0.90 & 2.21 & $3.086(4)$ & 163 \\
$\mathrm{~N} 4 A-\mathrm{H} 4 A 2 \cdots \mathrm{Cl} 2 A^{\text {iv }}$ & 0.90 & 2.56 & $3.405(2)$ & 157 \\
\hline
\end{tabular}

Symmetry codes: (i) $x+\frac{1}{2},-y+1, z+\frac{1}{2}$; (ii) $x-1, y, z$; (iii) $x-\frac{1}{2},-y+1, z-\frac{1}{2}$; (iv) $x+1, y, z$.

$\mathrm{Cr}$ (III) complex with 2,3,2-tet and additional $\mathrm{Cl}$ ligands, we report the structure of the title compound, trans- $\left[\mathrm{CrCl}_{2}(2,3,2-\right.$ tet) $\mathrm{ClO}_{4}$, (I), in this communication.

\section{Structural commentary}

Fig. 2 displays the molecular components of compound (I). In the distorted octahedral complex chromium(III) cation, the four $\mathrm{N}$ atoms of the 2,3,2-tet ligand occupy the equatorial sites and the two chlorine atoms coordinate axially to the metal. The two hydrogen atoms of the secondary amine groups are grouped on the same side (meso- $R S$ type) of the equatorial $\mathrm{N}_{4}$ plane. Such a conformation is consistent with those of trans$\left[\mathrm{CrF}_{2}(2,3,2\right.$-tet $\left.)\right] \mathrm{ClO}_{4}$ (Bang \& Pedersen, 1978) and trans$\left[\mathrm{Cr}(\mathrm{NCS})_{2}(2,3,2\right.$-tet)]NCS (Mäcke et al., 1982). The meso-RS conformation may be compared with $\mathrm{rac}$-RR/SS types of trans$\left[\mathrm{CrF}_{2}(2,2,3\right.$-tet $\left.)\right] \mathrm{ClO}_{4}$ (Choi \& Moon, 2014) and trans$\left[\mathrm{CrF}(3,2,3\right.$-tet $\left.)\left(\mathrm{H}_{2} \mathrm{O}\right)\right]\left(\mathrm{ClO}_{4}\right)_{2} \cdot \mathrm{H}_{2} \mathrm{O}$ (Choi \& Lee, 2008).

The $\mathrm{Cr}-\mathrm{N}$ bond lengths to the 2,3,2-tet ligand are in the range 2.069 (2) to 2.084 (2) $\AA$, in good agreement with those observed in the related structures of trans- $\left[\mathrm{CrF}_{2}(2,3,2-\right.$ tet) $\mathrm{ClO}_{4}$ (Bang \& Pedersen, 1978), trans-[Cr(NCS) 2 (2,3,2tet)]NCS (Mäcke et al., 1982), trans-[CrF 2 (2,2,3-tet) $] \mathrm{ClO}_{4}$ (Choi \& Moon, 2014), cis- $\beta$-[Cr(ox)(2,3,2-tet)]I (ox = oxalate; Kukina et al., 1990) and cis- $\beta-\left[\mathrm{Cr}\left(\mathrm{N}_{3}\right)_{2}(2,2,3-\right.$ tet $\left.)\right] \mathrm{Br}$ (Choi et al., 2011). The two $\mathrm{Cr}-\mathrm{Cl}$ distances in (I) average to 2.325 (2) $\AA$ and are close to the values found in cis- $\beta$ $\left[\mathrm{CrCl}_{2}(2,3,2\right.$-tet $\left.)\right] \mathrm{ClO}_{4}$ (Choi et al., 2008b) and cis- $\beta$ $\left[\mathrm{CrCl}_{2}\right.$ (2,2,3-tet) $] \mathrm{ClO}_{4}$ (Choi et al., 2008a). The $\mathrm{Cr} 1 A-\mathrm{N} 1 A$ and $\mathrm{Cr} 1 A-\mathrm{N} 4 A$ bond lengths to the primary amine $\mathrm{N}$ atoms are slightly longer than the $\operatorname{Cr} 1 A-\mathrm{N} 2 A$ and $\operatorname{Cr} 1 A-\mathrm{N} 3 A$ bond lengths to the secondary amine $\mathrm{N}$ atoms. It is interesting to note that the $\mathrm{Cr}-\mathrm{N}$ bond lengths to the primary amine $\mathrm{N}$ atoms in cis- $\beta$ - $\left[\mathrm{CrCl}_{2}(2,3,2\right.$-tet) $] \mathrm{ClO}_{4}$ (Choi et al., 2008b) are slightly shorter than those to the secondary amine $\mathrm{N}$ atoms. Two five-membered and one six-membered chelate rings of the 2,3,2-tet ligand are present in the structure of (I). They adopt gauche and stable chair conformations, respectively. The bond angles of the five- and six-membered chelate rings around the chromium(III) atom are $83.72(9)$ and $93.40(9)^{\circ}$, respectively. The other $\mathrm{N}-\mathrm{C}$ and $\mathrm{C}-\mathrm{C}$ bond lengths and $\mathrm{Cr}-$ $\mathrm{N}-\mathrm{C}, \mathrm{N}-\mathrm{C}-\mathrm{C}$ and $\mathrm{C}-\mathrm{C}-\mathrm{C}$ angles are normal for a 2,3,2tet ligand in a gauche or chair conformation. The tetrahedral $\mathrm{ClO}_{4}{ }^{-}$counter anion is distorted due to its involvement in hydrogen-bonding interactions. 


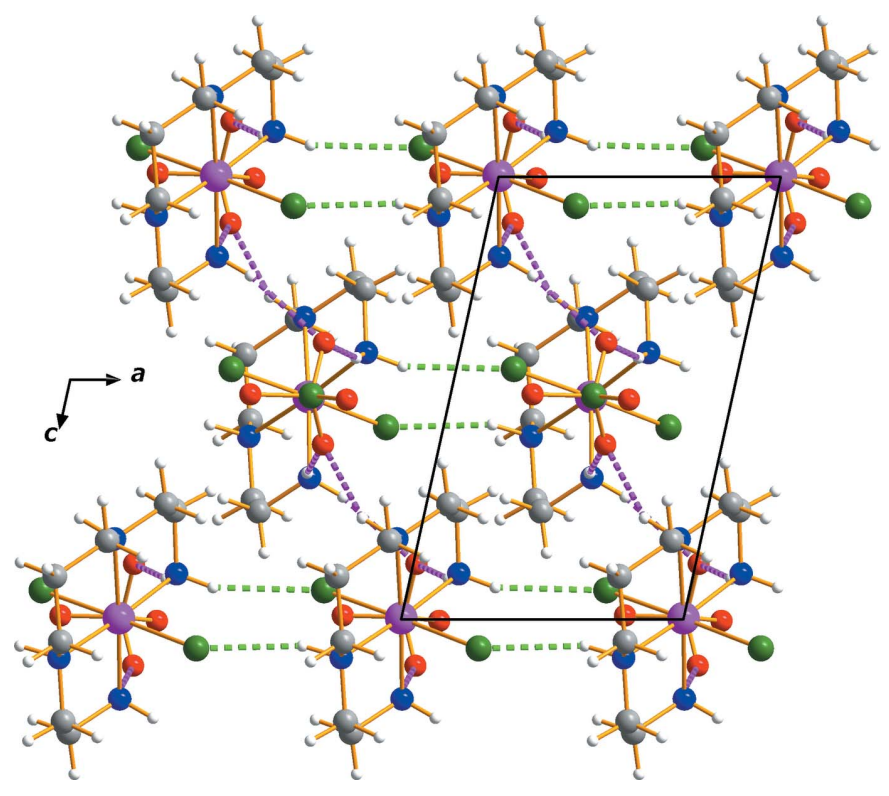

Figure 3

The crystal packing of complex (I) viewed perpendicular to (010). Dashed lines represent $\mathrm{N}-\mathrm{H} \cdots \mathrm{O}$ (pink) and $\mathrm{N}-\mathrm{H} \cdots \mathrm{Cl}$ (green) hydrogenbonding interactions, respectively.

\section{Supramolecular features}

In the crystal, molecules are stacked along [010]. An N$\mathrm{H} \cdots \mathrm{Cl}$ hydrogen bond ( $2 A \cdots \mathrm{Cl} 1 A)$ links neighboring cations into rows parallel to [100] while a series of $\mathrm{N}-\mathrm{H} \cdots \mathrm{O}$ contacts connect the cations to neighboring anions (Table 1). An extensive array of these contacts generates a two-dimensional network extending parallel to (010) (Figs. 3 and 4).

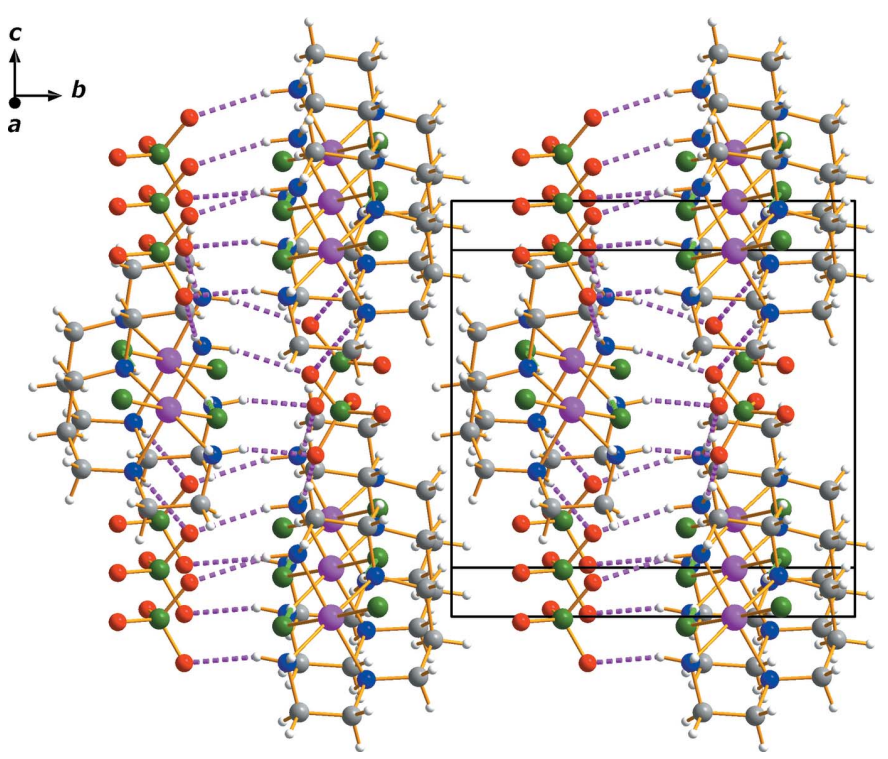

Figure 4

The crystal packing of complex (I) viewed approximately along [100]. The colour code is as in Fig. 3.

Table 2

Experimental details.

Crystal data

Chemical formula

$M_{\mathrm{r}}$

Crystal system, space group

Temperature (K)

$a, b, c(\AA)$

$\beta\left({ }^{\circ}\right)$

$V\left(\AA^{3}\right)$

$Z$

Radiation type

$\mu\left(\mathrm{mm}^{-1}\right)$

Crystal size (mm)

Data collection

Diffractometer

Absorption correction

$T_{\min }, T_{\max }$

No. of measured, independent and observed $[I>2 \sigma(I)]$ reflections

$R_{\text {int }}$

$(\sin \theta / \lambda)_{\max }\left(\AA^{-1}\right)$

Refinement

$R\left[F^{2}>2 \sigma\left(F^{2}\right)\right], w R\left(F^{2}\right), S$

No. of reflections

No. of parameters

No. of restraints

$\mathrm{H}$-atom treatment

$\Delta \rho_{\max }, \Delta \rho_{\min }\left(\mathrm{e} \AA^{-3}\right)$

Absolute structure

Absolute structure parameter

Computer programs: PAL BL2D-SMDC Program (Shin et al., 2016), HKL3000sm (Otwinowski \& Minor, 1997), SHELXT (Sheldrick, 2015a), SHELXL2014/7 (Sheldrick, 2015b), DIAMOND (Putz \& Brandenburg, 2014) and publCIF (Westrip, 2010).

\section{Database survey}

A search in the Cambridge Structural Database (Version 5.36, last update May 2015; Groom \& Allen, 2014) shows that there are four reports for $\mathrm{Cr}^{\mathrm{III}}$ complexes with a $\left[\mathrm{Cr} L_{2}(2,3,2 \text {-tet })\right]^{+}$ unit. The crystal structures of trans $-\left[\mathrm{CrF}_{2}(2,3,2\right.$-tet $\left.)\right] \mathrm{ClO}_{4}$ (Bang \& Pedersen, 1978), trans-[Cr(NCS $)_{2}(2,3,2$-tet) $] \mathrm{NCS}$ (Mäcke et al., 1982), cis- $\beta$-[Cr(ox)(2,3,2-tet)]I (Kukina et al., 1990), cis- $\beta-\left[\mathrm{CrCl}_{2}(2,3,2\right.$-tet $\left.)\right] \mathrm{ClO}_{4}$ (Choi et al., 2008b) have been reported previously. However, no structures of complexes of trans-[ $\mathrm{CrCl}_{2}(2,3,2$-tet $\left.)\right]^{+}$with any anions have been deposited.

\section{Synthesis and crystallization}

The free ligand 1,4,8,11-tetraazaundecane was purchased from Strem Chemical Company, USA. All other chemicals were reagent grade materials and were used without further purification. Compound (I) was prepared by a literature method (Kirk \& Fernando, 1994). The crude perchlorate salt (0.35 g) was dissolved in $20 \mathrm{~mL}$ of $0.1 \mathrm{M} \mathrm{HCl}$ at $333 \mathrm{~K}$. The filtrate was added to $5 \mathrm{~mL}$ of $60 \% \mathrm{HClO}_{4}$. The resulting solution was left 
for slow evaporation at room temperature. Green block-like crystals suitable for X-ray structural analysis were isolated after one week. The crystals were washed with small amounts of 2-propanol and dried in air before collecting the synchrotron data.

\section{Refinement}

Crystal data, data collection and structure refinement details are summarized in Table 2. The $\mathrm{H}$ atoms were placed in geometrically idealized positions and constrained to ride on their parent atoms, with $\mathrm{C}-\mathrm{H}$ distances of $0.98 \AA\left(\mathrm{C}-\mathrm{H}_{2}\right)$, and $\mathrm{N}-\mathrm{H}$ distances of $0.90 \AA$ and $0.99 \AA$ (secondary amine and primary amine $\mathrm{H}$ atoms, respectively), with $U_{\text {iso }}(\mathrm{H})$ values of $1.2 U_{\text {eq }}$ of the parent atoms.

\section{Acknowledgements}

This work was supported by a grant from 2016 Research Funds of Andong National University. The X-ray crystallography experiment at the PLS-II BL2D-SMC beamline was supported in part by MSIP and POSTECH.

\section{References}

Bang, E. \& Pedersen, E. (1978). Acta Chem. Scand. Ser. A, 32, 833836.

Choi, J.-H., Choi, S. Y., Hong, Y. P., Ko, S.-O., Ryoo, K. S., Lee, S. H. \& Park, Y. C. (2008a). Spectrochim. Acta Part A, 70, 619-625.

Choi, J.-H., Clegg, W. \& Harrington, R. W. (2011). Z. Anorg. Allg. Chem. 637, 562-566.
Choi, J.-H., Kim, H.-S. \& Habibi, M. H. (2008b). Bull. Korean Chem. Soc. 29, 1399-1402.

Choi, J.-H. \& Lee, U. (2008). Acta Cryst. E64, m1186.

Choi, J.-H. \& Moon, D. (2014). J. Mol. Struct. 1059, 325-331.

Groom, C. R. \& Allen, F. H. (2014). Angew. Chem. Int. Ed. 35, 31033111.

House, D. A. \& Garner, C. S. (1966). J. Am. Chem. Soc. 88, 21562162.

House, D. A. \& Yang, D. (1983). Inorg. Chim. Acta, 74, 179-189.

Kirk, A. D. \& Fernando, S. R. L. (1994). Inorg. Chem. 33, 44014047.

Kukina, G. A., Porai-Koshits, M. A., Shevchenko, Y. N. \& Shchurkina, V. N. (1990). Koord. Khim. 16, 784-792.

Kutal, C. \& Adamson, A. W. (1973). Inorg. Chem. 12, 1990-1994.

Mäcke, H. R., Mentzen, B. F., Puaux, J. P. \& Adamson, A. W. (1982). Inorg. Chem. 21, 3080-3082.

Otwinowski, Z. \& Minor, W. (1997). Methods in Enzymology, Vol. 276, Macromolecular Crystallography, Part A, edited by C. W. Carter Jr \& R. M. Sweet, pp. 307-326. Academic Press, New York.

Parsons, S., Flack, H. D. \& Wagner, T. (2013). Acta Cryst. B69, 249259.

Putz, H. \& Brandenburg, K. (2014). DIAMOND. Crystal Impact GbR, Bonn, Germany.

Ronconi, L. \& Sadler, P. J. (2007). Coord. Chem. Rev. 251, 16331648.

Ross, A., Choi, J.-H., Hunter, T. M., Pannecouque, C., Moggach, S. A., Parsons, S., De Clercq, E. \& Sadler, P. J. (2012). Dalton Trans. 41, 6408-6418.

Sheldrick, G. M. (2015a). Acta Cryst. A71, 3-8.

Sheldrick, G. M. (2015b). Acta Cryst. C71, 3-8.

Shin, J. W., Eom, K. \& Moon, D. (2016). J. Synchrotron Rad. 23, 369373.

Westrip, S. P. (2010). J. Appl. Cryst. 43, 920-925. 


\section{supporting information}

Acta Cryst. (2016). E72, 424-427 [doi:10.1107/S2056989016002978]

Crystal structure of trans-dichlorido(1,4,8,11-tetraazaundecane$\left.\kappa^{4} N\right)$ chromium(III) perchlorate determined from synchrotron data

\section{Dohyun Moon and Jong-Ha Choi}

Computing details

Data collection: PAL BL2D-SMDC Program (Shin et al., 2016); cell refinement: HKL3000sm (Otwinowski \& Minor, 1997); data reduction: HKL3000sm (Otwinowski \& Minor, 1997); program(s) used to solve structure: SHELXT (Sheldrick, 2015a); program(s) used to refine structure: SHELXL2014/7 (Sheldrick, 2015b); molecular graphics: DIAMOND (Putz \& Brandenburg, 2014); software used to prepare material for publication: publCIF (Westrip, 2010). trans-Dichlorido $\left(1,4,8,11\right.$-tetraazaundecane- $\left.\kappa^{4} N\right)$ chromium(III) perchlorate

Crystal data

$\left[\mathrm{CrCl}_{2}\left(\mathrm{C}_{7} \mathrm{H}_{20} \mathrm{~N}_{4}\right)\right] \mathrm{ClO}_{4}$

$M_{r}=382.62$

Monoclinic, $P n$

$a=6.4730(13) \AA$

$b=11.449$ (2) $\AA$

$c=10.385(2) \AA$

$\beta=102.42(3)^{\circ}$

$V=751.6(3) \AA^{3}$

$Z=2$

Data collection

ADSC Q210 CCD area-detector diffractometer

Radiation source: PLSII 2D bending magnet

$\omega$ scan

Absorption correction: empirical (using intensity measurements)

(HKL3000sm SCALEPACK; Otwinowski \& Minor, 1997)

$T_{\min }=0.893, T_{\max }=0.958$

Refinement

Refinement on $F^{2}$

Least-squares matrix: full

$R\left[F^{2}>2 \sigma\left(F^{2}\right)\right]=0.025$

$w R\left(F^{2}\right)=0.066$

$S=1.07$

4422 reflections

172 parameters

2 restraints
$F(000)=394$

$D_{\mathrm{x}}=1.691 \mathrm{Mg} \mathrm{m}^{-3}$

Synchrotron radiation, $\lambda=0.620 \AA$

Cell parameters from 22325 reflections

$\theta=0.4-33.6^{\circ}$

$\mu=0.89 \mathrm{~mm}^{-1}$

$T=243 \mathrm{~K}$

Block, green

$0.13 \times 0.13 \times 0.05 \mathrm{~mm}$

7831 measured reflections

4422 independent reflections

4214 reflections with $I>2 \sigma(I)$

$R_{\text {int }}=0.023$

$\theta_{\max }=26.0^{\circ}, \theta_{\min }=2.3^{\circ}$

$h=-9 \rightarrow 9$

$k=-16 \rightarrow 16$

$l=-14 \rightarrow 14$

Hydrogen site location: inferred from neighbouring sites

$\mathrm{H}$-atom parameters constrained

$w=1 /\left[\sigma^{2}\left(F_{\mathrm{o}}^{2}\right)+(0.0435 P)^{2}\right]$

where $P=\left(F_{\mathrm{o}}^{2}+2 F_{\mathrm{c}}^{2}\right) / 3$

$(\Delta / \sigma)_{\max }<0.001$

$\Delta \rho_{\max }=0.39 \mathrm{e} \AA^{-3}$

$\Delta \rho_{\min }=-0.62 \mathrm{e} \AA^{-3}$ 
Absolute structure: Flack $x$ determined using 2004 quotients $\left[\left(I^{+}\right)-\left(I^{\prime}\right)\right] /\left[\left(I^{+}\right)+\left(I^{-}\right)\right]$(Parsons et al., 2013)

Absolute structure parameter: 0.038 (9)

Special details

Geometry. All e.s.d.'s (except the e.s.d. in the dihedral angle between two 1.s. planes) are estimated using the full covariance matrix. The cell e.s.d.'s are taken into account individually in the estimation of e.s.d.'s in distances, angles and torsion angles; correlations between e.s.d.'s in cell parameters are only used when they are defined by crystal symmetry. An approximate (isotropic) treatment of cell e.s.d.'s is used for estimating e.s.d.'s involving 1.s. planes.

Fractional atomic coordinates and isotropic or equivalent isotropic displacement parameters $\left(\AA^{2}\right)$

\begin{tabular}{|c|c|c|c|c|}
\hline & $x$ & $y$ & $z$ & $U_{\text {iso }} * / U_{\text {eq }}$ \\
\hline $\mathrm{Cr} 1 \mathrm{~A}$ & $0.49958(5)$ & $0.29857(3)$ & 0.49729 (4) & $0.01583(8)$ \\
\hline Cl1A & $0.79710(9)$ & $0.18290(6)$ & $0.56558(7)$ & $0.02827(14)$ \\
\hline $\mathrm{Cl} 2 \mathrm{~A}$ & $0.20419(9)$ & $0.41841(5)$ & $0.43425(6)$ & $0.02410(12)$ \\
\hline N1A & $0.5655(3)$ & $0.3816(2)$ & $0.6797(2)$ & 0.0241 (4) \\
\hline H1A1 & 0.6982 & 0.3651 & 0.7229 & $0.029 *$ \\
\hline H1A2 & 0.5535 & 0.4595 & 0.6690 & $0.029 *$ \\
\hline N2A & $0.3179(3)$ & $0.18908(17)$ & $0.5865(2)$ & 0.0199 (4) \\
\hline $\mathrm{H} 2 \mathrm{~A}$ & 0.1708 & 0.2180 & 0.5598 & $0.024 *$ \\
\hline N3A & $0.4222(3)$ & $0.21127(19)$ & $0.3180(2)$ & $0.0234(4)$ \\
\hline $\mathrm{H} 3 \mathrm{~A}$ & 0.2837 & 0.2426 & 0.2713 & $0.028^{*}$ \\
\hline $\mathrm{N} 4 \mathrm{~A}$ & $0.6689(3)$ & $0.4076(2)$ & $0.3968(2)$ & $0.0249(4)$ \\
\hline H4A1 & 0.6368 & 0.4828 & 0.4087 & $0.030^{*}$ \\
\hline H4A2 & 0.8087 & 0.3978 & 0.4278 & $0.030 *$ \\
\hline $\mathrm{C} 1 \mathrm{~A}$ & $0.4110(5)$ & $0.3385(3)$ & $0.7556(3)$ & $0.0296(6)$ \\
\hline H1A3 & 0.2754 & 0.3789 & 0.7270 & $0.036^{*}$ \\
\hline $\mathrm{H} 1 \mathrm{~A} 4$ & 0.4636 & 0.3533 & 0.8499 & $0.036^{*}$ \\
\hline $\mathrm{C} 2 \mathrm{~A}$ & $0.3824(5)$ & $0.2085(3)$ & $0.7308(3)$ & $0.0284(5)$ \\
\hline $\mathrm{H} 2 \mathrm{~A} 1$ & 0.5152 & 0.1675 & 0.7664 & $0.034 *$ \\
\hline $\mathrm{H} 2 \mathrm{~A} 2$ & 0.2736 & 0.1783 & 0.7746 & $0.034 *$ \\
\hline $\mathrm{C} 3 \mathrm{~A}$ & $0.3107(4)$ & $0.0636(2)$ & $0.5507(3)$ & $0.0284(5)$ \\
\hline H3A1 & 0.2128 & 0.0230 & 0.5955 & $0.034 *$ \\
\hline $\mathrm{H} 3 \mathrm{~A} 2$ & 0.4515 & 0.0293 & 0.5812 & $0.034^{*}$ \\
\hline $\mathrm{C} 4 \mathrm{~A}$ & $0.2396(5)$ & $0.0455(3)$ & $0.4025(3)$ & $0.0335(6)$ \\
\hline $\mathrm{H} 4 \mathrm{~A} 3$ & 0.2074 & -0.0375 & 0.3859 & $0.040 *$ \\
\hline $\mathrm{H} 4 \mathrm{~A} 4$ & 0.1081 & 0.0892 & 0.3713 & $0.040^{*}$ \\
\hline C5A & $0.3975(5)$ & $0.0824(2)$ & $0.3219(3)$ & $0.0326(6)$ \\
\hline H5A1 & 0.5351 & 0.0471 & 0.3597 & $0.039 *$ \\
\hline H5A2 & 0.3510 & 0.0531 & 0.2317 & $0.039 *$ \\
\hline C6A & $0.5783(5)$ & $0.2472(3)$ & $0.2397(3)$ & $0.0333(6)$ \\
\hline H6A1 & 0.5258 & 0.2269 & 0.1467 & $0.040 *$ \\
\hline H6A2 & 0.7126 & 0.2064 & 0.2716 & $0.040 *$ \\
\hline $\mathrm{C} 7 \mathrm{~A}$ & $0.6117(5)$ & 0.3775 & $0.2536(3)$ & $0.0352(6)$ \\
\hline H7A1 & 0.7254 & 0.4016 & 0.2104 & $0.042 *$ \\
\hline H7A2 & 0.4821 & 0.4187 & 0.2113 & $0.042 *$ \\
\hline Cl1B & $0.50722(11)$ & $0.72486(5)$ & $0.49469(7)$ & $0.02745(12)$ \\
\hline
\end{tabular}




\begin{tabular}{lllll} 
O1B & $0.5868(6)$ & $0.6499(3)$ & $0.6047(3)$ & $0.0654(10)$ \\
O2B & $0.5109(4)$ & $0.6627(3)$ & $0.3746(3)$ & $0.0463(6)$ \\
O3B & $0.2904(5)$ & $0.7539(3)$ & $0.4912(3)$ & $0.0582(7)$ \\
O4B & $0.6361(5)$ & $0.8276(2)$ & $0.5033(3)$ & $0.0490(6)$ \\
\hline
\end{tabular}

Atomic displacement parameters $\left(\AA^{2}\right)$

\begin{tabular}{lllllll}
\hline & $U^{11}$ & $U^{22}$ & $U^{33}$ & $U^{12}$ & $U^{13}$ & $U^{23}$ \\
\hline Cr1A & $0.01154(13)$ & $0.01462(14)$ & $0.01988(14)$ & $0.00061(12)$ & $0.00019(10)$ & $-0.00054(13)$ \\
C11A & $0.0156(2)$ & $0.0267(3)$ & $0.0401(3)$ & $0.0062(2)$ & $0.0006(2)$ & $0.0027(2)$ \\
C12A & $0.0175(2)$ & $0.0225(3)$ & $0.0308(3)$ & $0.00531(19)$ & $0.00184(18)$ & $0.0056(2)$ \\
N1A & $0.0234(9)$ & $0.0212(10)$ & $0.0244(9)$ & $0.0001(8)$ & $-0.0019(7)$ & $-0.0040(8)$ \\
N2A & $0.0163(8)$ & $0.0153(9)$ & $0.0270(10)$ & $-0.0001(7)$ & $0.0018(7)$ & $0.0032(7)$ \\
N3A & $0.0242(10)$ & $0.0211(10)$ & $0.0237(10)$ & $-0.0005(8)$ & $0.0024(8)$ & $-0.0044(8)$ \\
N4A & $0.0186(9)$ & $0.0227(11)$ & $0.0337(11)$ & $-0.0008(7)$ & $0.0063(8)$ & $0.0026(8)$ \\
C1A & $0.0364(14)$ & $0.0305(15)$ & $0.0222(11)$ & $0.0035(11)$ & $0.0071(10)$ & $-0.0034(10)$ \\
C2A & $0.0369(15)$ & $0.0236(13)$ & $0.0248(12)$ & $0.0033(10)$ & $0.0072(10)$ & $0.0056(9)$ \\
C3A & $0.0293(12)$ & $0.0156(11)$ & $0.0388(13)$ & $-0.0013(9)$ & $0.0037(10)$ & $0.0025(10)$ \\
C4A & $0.0333(14)$ & $0.0204(12)$ & $0.0422(15)$ & $-0.0079(10)$ & $-0.0023(11)$ & $-0.0055(11)$ \\
C5A & $0.0386(15)$ & $0.0210(13)$ & $0.0358(13)$ & $-0.0006(10)$ & $0.0025(11)$ & $-0.0097(10)$ \\
C6A & $0.0362(15)$ & $0.0376(18)$ & $0.0283(13)$ & $0.0012(12)$ & $0.0118(11)$ & $-0.0037(12)$ \\
C7A & $0.0369(15)$ & $0.0399(18)$ & $0.0316(13)$ & $-0.0016(13)$ & $0.0133(11)$ & $0.0052(12)$ \\
C11B & $0.0312(3)$ & $0.0245(3)$ & $0.0255(2)$ & $0.0024(3)$ & $0.00349(19)$ & $0.0028(3)$ \\
O1B & $0.077(2)$ & $0.0510(16)$ & $0.0495(15)$ & $-0.0175(15)$ & $-0.0279(14)$ & $0.0247(13)$ \\
O2B & $0.0544(16)$ & $0.0478(14)$ & $0.0396(12)$ & $-0.0064(12)$ & $0.0165(11)$ & $-0.0106(10)$ \\
O3B & $0.0421(14)$ & $0.063(2)$ & $0.075(2)$ & $0.0116(13)$ & $0.0243(13)$ & $0.0017(16)$ \\
O4B & $0.0587(17)$ & $0.0279(12)$ & $0.0574(15)$ & $-0.0124(11)$ & $0.0060(12)$ & $0.0005(10)$ \\
& & & & & &
\end{tabular}

Geometric parameters $\left(\AA,^{\circ}\right)$

\begin{tabular}{llll}
\hline Cr1A-N2A & $2.069(2)$ & $\mathrm{C} 1 \mathrm{~A}-\mathrm{H} 1 \mathrm{~A} 4$ & 0.9800 \\
$\mathrm{Cr} 1 \mathrm{~A}-\mathrm{N} 3 \mathrm{~A}$ & $2.078(2)$ & $\mathrm{C} 2 \mathrm{~A}-\mathrm{H} 2 \mathrm{~A} 1$ & 0.9800 \\
$\mathrm{Cr} 1 \mathrm{~A}-\mathrm{N} 1 \mathrm{~A}$ & $2.080(2)$ & $\mathrm{C} 2 \mathrm{~A}-\mathrm{H} 2 \mathrm{~A} 2$ & 0.9800 \\
$\mathrm{Cr} 1 \mathrm{~A}-\mathrm{N} 4 \mathrm{~A}$ & $2.084(2)$ & $\mathrm{C} 3 \mathrm{~A}-\mathrm{C} 4 \mathrm{~A}$ & $1.523(4)$ \\
$\mathrm{Cr} 1 \mathrm{~A}-\mathrm{C} 11 \mathrm{~A}$ & $2.3191(8)$ & $\mathrm{C} 3 \mathrm{~A}-\mathrm{H} 3 \mathrm{~A} 1$ & 0.9800 \\
$\mathrm{Cr} 1 \mathrm{~A}-\mathrm{C} 2 \mathrm{~A}$ & $2.3300(8)$ & $\mathrm{C} 3 \mathrm{~A}-\mathrm{H} 3 \mathrm{~A} 2$ & 0.9800 \\
$\mathrm{~N} 1 \mathrm{~A}-\mathrm{C} 1 \mathrm{~A}$ & $1.484(4)$ & $\mathrm{C} 4 \mathrm{~A}-\mathrm{C} 5 \mathrm{~A}$ & $1.514(4)$ \\
$\mathrm{N} 1 \mathrm{~A}-\mathrm{H} 1 \mathrm{~A} 1$ & 0.9000 & $\mathrm{C} 4 \mathrm{~A}-\mathrm{H} 4 \mathrm{~A} 3$ & 0.9800 \\
$\mathrm{~N} 1 \mathrm{~A}-\mathrm{H} 1 \mathrm{~A} 2$ & 0.9000 & $\mathrm{C} 4 \mathrm{~A}-\mathrm{H} 4 \mathrm{~A} 4$ & 0.9800 \\
$\mathrm{~N} 2 \mathrm{~A}-\mathrm{C} 3 \mathrm{~A}$ & $1.482(3)$ & $\mathrm{C} 5 \mathrm{~A}-\mathrm{H} 5 \mathrm{~A} 1$ & 0.9800 \\
$\mathrm{~N} 2 \mathrm{~A}-\mathrm{C} 2 \mathrm{~A}$ & $1.483(4)$ & $\mathrm{C} 5 \mathrm{~A}-\mathrm{H} 5 \mathrm{~A} 2$ & 0.9800 \\
$\mathrm{~N} 2 \mathrm{~A}-\mathrm{H} 2 \mathrm{~A}$ & 0.9900 & $\mathrm{C} 6 \mathrm{~A}-\mathrm{C} 7 \mathrm{~A}$ & $1.510(5)$ \\
$\mathrm{N} 3 \mathrm{~A}-\mathrm{C} 5 \mathrm{~A}$ & $1.485(3)$ & $\mathrm{C} 6 \mathrm{~A}-\mathrm{H} 6 \mathrm{~A} 1$ & 0.9800 \\
$\mathrm{~N} 3 \mathrm{~A}-\mathrm{C} 6 \mathrm{~A}$ & $1.485(4)$ & $\mathrm{C} 6 \mathrm{~A}-\mathrm{H} 6 \mathrm{~A} 2$ & 0.9800 \\
$\mathrm{~N} 3 \mathrm{~A}-\mathrm{H} 3 \mathrm{~A}$ & 0.9900 & $\mathrm{C} 7 \mathrm{~A}-\mathrm{H} 7 \mathrm{~A} 1$ & 0.9800 \\
$\mathrm{~N} 4 \mathrm{~A}-\mathrm{C} 7 \mathrm{~A}$ & $1.493(4)$ & $\mathrm{C} 7 \mathrm{~A}-\mathrm{H} 7 \mathrm{~A} 2$ & 0.9800 \\
$\mathrm{~N} 4 \mathrm{~A}-\mathrm{H} 4 \mathrm{~A} 1$ & 0.9000 & $\mathrm{Cl1B}-\mathrm{O} 1 \mathrm{~B}$ & $1.433(3)$ \\
$\mathrm{N} 4 \mathrm{~A}-\mathrm{H} 4 \mathrm{~A} 2$ & 0.9000 & $\mathrm{Cl} 1 \mathrm{~B}-\mathrm{O} 4 \mathrm{~B}$ & $1.434(2)$
\end{tabular}


$\mathrm{C} 1 \mathrm{~A}-\mathrm{C} 2 \mathrm{~A}$

$\mathrm{C} 1 \mathrm{~A}-\mathrm{H} 1 \mathrm{~A} 3$

$\mathrm{N} 2 \mathrm{~A}-\mathrm{Cr} 1 \mathrm{~A}-\mathrm{N} 3 \mathrm{~A}$

$\mathrm{N} 2 \mathrm{~A}-\mathrm{Cr} 1 \mathrm{~A}-\mathrm{N} 1 \mathrm{~A}$

N3A-Cr1A-N1A

$\mathrm{N} 2 \mathrm{~A}-\mathrm{Cr} 1 \mathrm{~A}-\mathrm{N} 4 \mathrm{~A}$

N3A-Cr1A-N4A

N1A-Cr1A-N4A

$\mathrm{N} 2 \mathrm{~A}-\mathrm{Cr} 1 \mathrm{~A}-\mathrm{C} 11 \mathrm{~A}$

N3A-Cr1A-Cl1A

N1A-Cr1A-Cl1A

N4A-Cr1A-Cl1A

$\mathrm{N} 2 \mathrm{~A}-\mathrm{Cr} 1 \mathrm{~A}-\mathrm{Cl} 2 \mathrm{~A}$

N3A-Cr1A-Cl2A

N1A-Cr1A-Cl2A

$\mathrm{N} 4 \mathrm{~A}-\mathrm{Cr} 1 \mathrm{~A}-\mathrm{Cl} 2 \mathrm{~A}$

$\mathrm{Cl} 1 \mathrm{~A}-\mathrm{Cr} 1 \mathrm{~A}-\mathrm{Cl} 2 \mathrm{~A}$

C1A-N1A-Cr1A

C1A-N1A-H1A1

Cr1A-N1A-H1A1

C1A-N1A-H1A2

Cr1A-N1A-H1A2

H1A1-N1A-H1A2

$\mathrm{C} 3 \mathrm{~A}-\mathrm{N} 2 \mathrm{~A}-\mathrm{C} 2 \mathrm{~A}$

$\mathrm{C} 3 \mathrm{~A}-\mathrm{N} 2 \mathrm{~A}-\mathrm{Cr} 1 \mathrm{~A}$

$\mathrm{C} 2 \mathrm{~A}-\mathrm{N} 2 \mathrm{~A}-\mathrm{Cr} 1 \mathrm{~A}$

$\mathrm{C} 3 \mathrm{~A}-\mathrm{N} 2 \mathrm{~A}-\mathrm{H} 2 \mathrm{~A}$

$\mathrm{C} 2 \mathrm{~A}-\mathrm{N} 2 \mathrm{~A}-\mathrm{H} 2 \mathrm{~A}$

Cr1A-N2A-H2A

$\mathrm{C} 5 \mathrm{~A}-\mathrm{N} 3 \mathrm{~A}-\mathrm{C} 6 \mathrm{~A}$

$\mathrm{C} 5 \mathrm{~A}-\mathrm{N} 3 \mathrm{~A}-\mathrm{Cr} 1 \mathrm{~A}$

C6A-N3A-Cr1A

$\mathrm{C} 5 \mathrm{~A}-\mathrm{N} 3 \mathrm{~A}-\mathrm{H} 3 \mathrm{~A}$

C6A-N3A-H3A

$\mathrm{Cr} 1 \mathrm{~A}-\mathrm{N} 3 \mathrm{~A}-\mathrm{H} 3 \mathrm{~A}$

C7A-N4A-Cr1A

C7A-N4A-H4A1

$\mathrm{Cr} 1 \mathrm{~A}-\mathrm{N} 4 \mathrm{~A}-\mathrm{H} 4 \mathrm{~A} 1$

$\mathrm{C} 7 \mathrm{~A}-\mathrm{N} 4 \mathrm{~A}-\mathrm{H} 4 \mathrm{~A} 2$

$\mathrm{Cr} 1 \mathrm{~A}-\mathrm{N} 4 \mathrm{~A}-\mathrm{H} 4 \mathrm{~A} 2$

$\mathrm{H} 4 \mathrm{~A} 1-\mathrm{N} 4 \mathrm{~A}-\mathrm{H} 4 \mathrm{~A} 2$

$\mathrm{N} 1 \mathrm{~A}-\mathrm{C} 1 \mathrm{~A}-\mathrm{C} 2 \mathrm{~A}$

$\mathrm{N} 1 \mathrm{~A}-\mathrm{C} 1 \mathrm{~A}-\mathrm{H} 1 \mathrm{~A} 3$

$\mathrm{C} 2 \mathrm{~A}-\mathrm{C} 1 \mathrm{~A}-\mathrm{H} 1 \mathrm{~A} 3$

$\mathrm{N} 1 \mathrm{~A}-\mathrm{C} 1 \mathrm{~A}-\mathrm{H} 1 \mathrm{~A} 4$

$\mathrm{C} 2 \mathrm{~A}-\mathrm{C} 1 \mathrm{~A}-\mathrm{H} 1 \mathrm{~A} 4$
$1.514(4)$

0.9800

$93.40(9)$

83.91 (9)

$177.24(9)$

$176.52(9)$

$83.72(9)$

98.95 (9)

$91.81(6)$

$91.36(7)$

$89.33(7)$

$90.21(7)$

88.37 (6)

$90.36(7)$

$88.97(7)$

89.69 (7)

$178.26(3)$

$107.58(16)$

110.2

110.2

110.2

110.2

108.5

112.7 (2)

$117.73(18)$

107.34 (16)

106.1

106.1

106.1

112.4 (2)

$117.36(17)$

$107.22(17)$

106.4

106.4

106.4

$108.43(17)$

110.0

110.0

110.0

110.0

108.4

$108.0(2)$

110.1

110.1

110.1

110.1
$\mathrm{C} 11 \mathrm{~B}-\mathrm{O} 3 \mathrm{~B}$

$\mathrm{C} 11 \mathrm{~B}-\mathrm{O} 2 \mathrm{~B}$

$1.435(3)$

$1.441(3)$

$\begin{array}{ll}\mathrm{H} 1 \mathrm{~A} 3-\mathrm{C} 1 \mathrm{~A}-\mathrm{H} 1 \mathrm{~A} 4 & 108.4 \\ \mathrm{~N} 2 \mathrm{~A}-\mathrm{C} 2 \mathrm{~A}-\mathrm{C} 1 \mathrm{~A} & 108.5(2)\end{array}$

$\mathrm{N} 2 \mathrm{~A}-\mathrm{C} 2 \mathrm{~A}-\mathrm{H} 2 \mathrm{~A} 1 \quad 110.0$

$\mathrm{C} 1 \mathrm{~A}-\mathrm{C} 2 \mathrm{~A}-\mathrm{H} 2 \mathrm{~A} 1 \quad 110.0$

$\mathrm{N} 2 \mathrm{~A}-\mathrm{C} 2 \mathrm{~A}-\mathrm{H} 2 \mathrm{~A} 2 \quad 110.0$

$\mathrm{C} 1 \mathrm{~A}-\mathrm{C} 2 \mathrm{~A}-\mathrm{H} 2 \mathrm{~A} 2 \quad 110.0$

$\mathrm{H} 2 \mathrm{~A} 1-\mathrm{C} 2 \mathrm{~A}-\mathrm{H} 2 \mathrm{~A} 2 \quad 108.4$

$\mathrm{N} 2 \mathrm{~A}-\mathrm{C} 3 \mathrm{~A}-\mathrm{C} 4 \mathrm{~A} \quad 111.8(2)$

$\mathrm{N} 2 \mathrm{~A}-\mathrm{C} 3 \mathrm{~A}-\mathrm{H} 3 \mathrm{~A} 1 \quad 109.3$

C4A-C3A-H3A1 109.3

$\mathrm{N} 2 \mathrm{~A}-\mathrm{C} 3 \mathrm{~A}-\mathrm{H} 3 \mathrm{~A} 2 \quad 109.3$

$\mathrm{C} 4 \mathrm{~A}-\mathrm{C} 3 \mathrm{~A}-\mathrm{H} 3 \mathrm{~A} 2 \quad 109.3$

$\mathrm{H} 3 \mathrm{~A} 1-\mathrm{C} 3 \mathrm{~A}-\mathrm{H} 3 \mathrm{~A} 2 \quad 107.9$

$\mathrm{C} 5 \mathrm{~A}-\mathrm{C} 4 \mathrm{~A}-\mathrm{C} 3 \mathrm{~A} \quad 115.3$ (2)

$\mathrm{C} 5 \mathrm{~A}-\mathrm{C} 4 \mathrm{~A}-\mathrm{H} 4 \mathrm{~A} 3 \quad 108.5$

$\mathrm{C} 3 \mathrm{~A}-\mathrm{C} 4 \mathrm{~A}-\mathrm{H} 4 \mathrm{~A} 3 \quad 108.5$

$\mathrm{C} 5 \mathrm{~A}-\mathrm{C} 4 \mathrm{~A}-\mathrm{H} 4 \mathrm{~A} 4 \quad 108.5$

$\mathrm{C} 3 \mathrm{~A}-\mathrm{C} 4 \mathrm{~A}-\mathrm{H} 4 \mathrm{~A} 4 \quad 108.5$

$\mathrm{H} 4 \mathrm{~A} 3-\mathrm{C} 4 \mathrm{~A}-\mathrm{H} 4 \mathrm{~A} 4 \quad 107.5$

$\mathrm{N} 3 \mathrm{~A}-\mathrm{C} 5 \mathrm{~A}-\mathrm{C} 4 \mathrm{~A} \quad 112.5$ (2)

N3A-C5A-H5A1 109.1

C4A-C5A-H5A1 109.1

$\mathrm{N} 3 \mathrm{~A}-\mathrm{C} 5 \mathrm{~A}-\mathrm{H} 5 \mathrm{~A} 2 \quad 109.1$

$\mathrm{C} 4 \mathrm{~A}-\mathrm{C} 5 \mathrm{~A}-\mathrm{H} 5 \mathrm{~A} 2 \quad 109.1$

$\mathrm{H} 5 \mathrm{~A} 1-\mathrm{C} 5 \mathrm{~A}-\mathrm{H} 5 \mathrm{~A} 2 \quad 107.8$

N3A-C6A-C7A 108.8 (2)

N3A-C6A-H6A1 109.9

C7A-C6A-H6A1 109.9

N3A-C6A-H6A2 109.9

C7A-C6A-H6A2 109.9

H6A1-C6A-H6A2 108.3

$\mathrm{N} 4 \mathrm{~A}-\mathrm{C} 7 \mathrm{~A}-\mathrm{C} 6 \mathrm{~A} \quad 108.8$ (2)

N4A-C7A-H7A1 109.9

C6A-C7A-H7A1 109.9

$\mathrm{N} 4 \mathrm{~A}-\mathrm{C} 7 \mathrm{~A}-\mathrm{H} 7 \mathrm{~A} 2 \quad 109.9$

$\mathrm{C} 6 \mathrm{~A}-\mathrm{C} 7 \mathrm{~A}-\mathrm{H} 7 \mathrm{~A} 2 \quad 109.9$

H7A1-C7A-H7A2 108.3

O1B-Cl1B-O4B 109.70 (17)

$\mathrm{O} 1 \mathrm{~B}-\mathrm{Cl1B}-\mathrm{O} 3 \mathrm{~B} \quad 109.9(2)$

$\mathrm{O} 4 \mathrm{~B}-\mathrm{Cl1B}-\mathrm{O} 3 \mathrm{~B} \quad 111.3(2)$

$\mathrm{O} 1 \mathrm{~B}-\mathrm{C} 11 \mathrm{~B}-\mathrm{O} 2 \mathrm{~B} \quad 108.91$ (19)

$\mathrm{O} 4 \mathrm{~B}-\mathrm{Cl1B}-\mathrm{O} 2 \mathrm{~B} \quad 109.98(17)$

$\mathrm{O} 3 \mathrm{~B}-\mathrm{Cl1B}-\mathrm{O} 2 \mathrm{~B} \quad 106.93(19)$ 


$\begin{array}{llll}\mathrm{Cr} 1 \mathrm{~A}-\mathrm{N} 1 \mathrm{~A}-\mathrm{C} 1 \mathrm{~A}-\mathrm{C} 2 \mathrm{~A} & 40.4(2) & \mathrm{C} 6 \mathrm{~A}-\mathrm{N} 3 \mathrm{~A}-\mathrm{C} 5 \mathrm{~A}-\mathrm{C} 4 \mathrm{~A} & 179.6(2) \\ \mathrm{C} 3 \mathrm{~A}-\mathrm{N} 2 \mathrm{~A}-\mathrm{C} 2 \mathrm{~A}-\mathrm{C} 1 \mathrm{~A} & 173.1(2) & \mathrm{C} 1 \mathrm{~A}-\mathrm{N} 3 \mathrm{~A}-\mathrm{C} 5 \mathrm{~A}-\mathrm{C} 4 \mathrm{~A} & 54.6(3) \\ \mathrm{Cr} 1 \mathrm{~A}-\mathrm{N} 2 \mathrm{~A}-\mathrm{C} 2 \mathrm{~A}-\mathrm{C} 1 \mathrm{~A} & 41.9(3) & \mathrm{C} 3 \mathrm{~A}-\mathrm{C} 4 \mathrm{~A}-\mathrm{C} 5 \mathrm{~A}-\mathrm{N} 3 \mathrm{~A} & -70.3(3) \\ \mathrm{N} 1 \mathrm{~A}-\mathrm{C} 1 \mathrm{~A}-\mathrm{C} 2 \mathrm{~A}-\mathrm{N} 2 \mathrm{~A} & -55.7(3) & \mathrm{C} 5 \mathrm{~A}-\mathrm{N} 3 \mathrm{~A}-\mathrm{C} 6 \mathrm{~A}-\mathrm{C} 7 \mathrm{~A} & -173.7(2) \\ \mathrm{C} 2 \mathrm{~A}-\mathrm{N} 2 \mathrm{~A}-\mathrm{C} 3 \mathrm{~A}-\mathrm{C} 4 \mathrm{~A} & 178.8(2) & \mathrm{Cr} 1 \mathrm{~A}-\mathrm{N} 3 \mathrm{~A}-\mathrm{C} 6 \mathrm{~A}-\mathrm{C} 7 \mathrm{~A} & -43.3(3) \\ \mathrm{Cr} 1 \mathrm{~A}-\mathrm{N} 2 \mathrm{~A}-\mathrm{C} 3 \mathrm{~A}-\mathrm{C} 4 \mathrm{~A} & -55.5(3) & \mathrm{Cr} 1 \mathrm{~A}-\mathrm{N} 4 \mathrm{~A}-\mathrm{C} 7 \mathrm{~A}-\mathrm{C} 6 \mathrm{~A} & -36.0(3) \\ \mathrm{N} 2 \mathrm{~A}-\mathrm{C} 3 \mathrm{~A}-\mathrm{C} 4 \mathrm{~A}-\mathrm{C} 5 \mathrm{~A} & 70.5(3) & \mathrm{N} 3 \mathrm{~A}-\mathrm{C} 6 \mathrm{~A}-\mathrm{C} 7 \mathrm{~A}-\mathrm{N} 4 \mathrm{~A} & 53.5(3)\end{array}$

Hydrogen-bond geometry $\left(A,{ }^{\circ}\right)$

\begin{tabular}{lllll}
\hline$D-\mathrm{H} \cdots A$ & $D-\mathrm{H}$ & $\mathrm{H} \cdots A$ & $D \cdots A$ & $D-\mathrm{H} \cdots A$ \\
\hline $\mathrm{N} 1 A-\mathrm{H} 1 A 1 \cdots \mathrm{O} 2 B^{\mathrm{i}}$ & 0.90 & 2.30 & $3.187(4)$ & 167 \\
$\mathrm{~N} 1 A-\mathrm{H} 1 A 2 \cdots \mathrm{O} 1 B$ & 0.90 & 2.30 & $3.180(4)$ & 164 \\
$\mathrm{~N} 2 A-\mathrm{H} 2 A \cdots \mathrm{C} 11 A^{\mathrm{ii}}$ & 0.99 & 2.47 & $3.332(2)$ & 146 \\
$\mathrm{~N} 3 A-\mathrm{H} 3 A \cdots \mathrm{O} 1 B^{\text {iii }}$ & 0.99 & 2.28 & $3.174(4)$ & 150 \\
$\mathrm{~N} 4 A-\mathrm{H} 4 A 1 \cdots \mathrm{O} 2 B$ & 0.90 & 2.21 & $3.086(4)$ & 163 \\
$\mathrm{~N} 4 A-\mathrm{H} 4 A 2 \cdots \mathrm{Cl} 2 A^{\mathrm{iv}}$ & 0.90 & 2.56 & $3.405(2)$ & 157
\end{tabular}

Symmetry codes: (i) $x+1 / 2,-y+1, z+1 / 2$; (ii) $x-1, y, z$; (iii) $x-1 / 2,-y+1, z-1 / 2$; (iv) $x+1, y, z$. 